\title{
Ethyl Alcohol Mouthwash
}

National Cancer Institute

\section{Source}

National Cancer Institute. Ethyl Alcohol Mouthwash. NCI Thesaurus. Code C121781.

A mouthwash containing ethyl alcohol, with topical antibacterial activity. Upon rinsing the oral cavity with the ethyl alcohol mouthwash, ethyl alcohol denatures bacterial proteins, and dissolves and disrupts the bacterial lipid membrane, thereby killing the bacteria. This reduces oral colonization with harmful bacteria and may prevent oral mucositis. 